\title{
Posterior Lateral Meniscal Root Tears Increase Strain on the Reconstructed Anterior Cruciate Ligament: A Cadaveric Study
}

\author{
William Uffmann, M.D., Neal ElAttrache, M.D., Trevor Nelson, B.S., Sam A. Eberlein, B.S., \\ Juntian Wang, M.D., Daniel R. Howard, M.D., and Melodie F. Metzger, M.D.
}

\begin{abstract}
Purpose: To quantify the amount of strain across an anterior cruciate ligament reconstruction (ACLR) before and after a lateral meniscus (LM) posterior root complex tear and determine whether a meniscal root repair effectively protects the ACLR against excessive strain. Methods: Fresh-frozen cadaveric knees were tested with an $88-\mathrm{N}$ anterior drawer force and an internal and external torque of $5-\mathrm{Nm}$ applied at $0^{\circ}, 15^{\circ}, 30^{\circ}, 60^{\circ}$, and $90^{\circ}$ of flexion. A simulated pivot shift was also applied at 0,15 , and $30^{\circ}$ of flexion. Rotation and translation of the tibia, and strain across the ACL graft were recorded. Testing was repeated for the following four conditions: ACL-intact, ACLR with intact LM, ACLR with LM posterior root complex tear, and ACLR with root repair. Results: The kinematic data from 12 fresh frozen cadaveric knees underwent analysis. Only 11 specimens had usable strain data. Sectioning the meniscofemoral ligaments and the LM posterior root increased rotational and translational laxity at $30^{\circ}$ of knee flexion. ACLR graft strain significantly increased when an anterior load and internal torque were applied. Repair of the LM posterior root reduced strain when the knee was internally rotated but was unable to normalize strain when an anterior force was applied. Conclusions: This cadaveric biomechanical study suggests injury to the LM posterior root complex increases rotational and anterior laxity of the knee and places increased strain across reconstructed ACL grafts. Subsequent root repair did not result in a statistically significant reduction in strain. Clinical Relevance: This study provides quantitative data on the implications of a LM posterior root injury in the setting of an ACL reconstruction to help guide clinical decision-making.
\end{abstract}

$\mathbf{L}^{2}$ ateral meniscus (LM) posterior root tears are prevalent injuries, occurring in $8 \%$ to $14 \%$ of patients with concomitant anterior cruciate ligament

From Cedars-Sinai Kerlan-Jobe Institute (W.U., N.E.); and Orthopaedic Biomechanics Laboratory (T.N., S.A.E., M.F.M.) and Department of Orthopaedic Surgery (J.W., M.F.M.), Cedars-Sinai Medical Center, Los Angeles, California; and Somers Orthopaedic Surgery e Sports Medicine, Carmel, New York (D.R.H.), U.S.A.

The authors report the following potential conflicts of interest or sources of funding: D.R.H. reports grants from DJO, Arthrex, and Smith $\theta$ Nephew, outside the submitted work. N.E. reports grants from Arthrex, during the conduct of the study; and personal fees and nonfinancial support from Arthrex, outside the submitted work. W.U. reports grants from Arthrex, during the conduct of the study; and grants and other from Arthrex, outside the submitted work. This research was funded by a generous research grant by Arthrex (RDGT0410). Full ICMJE author disclosure forms are available for this article online, as supplementary material.

Received November 30, 2019; accepted November 25, 2020.

Address correspondence to William Uffmann, M.D., Essentia Health Duluth Clinic, 400 E. 3rd St., Duluth, MN 55805. E-mail: william. uffmann@gmail.com

(C) 2020 THE AUTHORS. Published by Elsevier Inc. on behalf of the Arthroscopy Association of North America. This is an open access article under the CC BY-NC-ND license (http://creativecommons.org/licenses/by-nc-nd/4.0/). 2666-061X/191419

https://doi.org/10.1016/j.asmr.2020.11.005
(ACL) injuries. ${ }^{1-6}$ Meniscal root tears have been compared with subtotal meniscectomies in terms of their ability to alter joint loads and contact stresses. ${ }^{6-10}$ In addition, root tears are associated with meniscal extrusion, ${ }^{8,11}$ rotational instability, ${ }^{6-10}$ and have been linked to degenerative changes in the knee. ${ }^{10,12}$

Previous biomechanical studies demonstrate that the LM posterior root helps stabilize the knee against excessive anterior tibial translation in lower flexion angles and against internal rotation in greater flexion angles, ${ }^{6,10}$ and, when torn, contributes to further translational and rotational instability in ACL-deficient knees. ${ }^{6,7,9}$ Furthermore, recent in vitro studies suggest anatomic root repair can normalize tibiofemoral contact pressures $^{13-15}$ and restore native kinematics in both an ACL-deficient ${ }^{6}$ and concomitant anterior cruciate ligament reconstruction (ACLR) setting. ${ }^{7}$ Thus, menisci have been regarded as important secondary stabilizers of the knee and their functional absence is thought to jeopardize the outcome of concurrent ligament reconstructions, including the ACL. This study directly evaluated the effect of LM posterior root tears on strain across an ACL reconstruction graft. 
The purpose of this study was to quantify the amount of strain across an ACLR before and after a LM posterior root complex tear and determine whether a meniscal root repair effectively protects the ACLR against excessive strain. It is hypothesized that meniscal root injury will place increased strain upon the reconstructed ACL graft and that meniscal root repair will decrease this increase in strain.

\section{Methods}

\section{Specimen Preparation}

Paired male and female specimens aged 18 to 60 years were included. Specimens were visually examined by a sports fellowship-trained orthopaedic surgeon (W.J.U.) and evaluated for suitability and to ensure no varus or valgus deformity, ligamentous laxity, any visible arthritic wear, previous surgical history, or gross anatomic abnormality were present. Specimens were stored at $-20^{\circ} \mathrm{C}$ and thawed at room temperature 24 hours before testing. The femoral diaphysis and tibial diaphysis were sectioned 20 and $15 \mathrm{~cm}$ from the joint line, respectively. The muscle bellies of the quadriceps and hamstrings were sectioned $5 \mathrm{~cm}$ distal to the femoral osteotomy preserving their tendinous insertions and the skin overlying the knee. Similarly, the muscles of the 4 compartments of the lower leg were sectioned $5 \mathrm{~cm}$ proximal to the tibial osteotomy preserving any proximal skin and all ligamentous and tendinous peri-articular origins. The distal portion of the tibia was potted in a custom machined aluminum cylinder.

A median parapatellar arthrotomy was made to facilitate inspection of the specimen and allow reproducible placement of the strain gauge. Before testing all ACLR states, the specimen was prepared for the placement of a strain gauge. Placement of the differential variable reluctance transducer (DVRT) strain gauge was carried out in a similar manner as outlined by Mancini et al. ${ }^{16}$ A standardized 5 -mm notchplasty was made at the superolateral intercondylar notch on all specimens using a 5-mm round burr to prevent impingement of the DVRT sensor with the femur. For each specimen, the strain gauge (3-mm stroke microminiature DVRT; Lord Corp., Williston, VT) was placed within the mid-substance of the ACL or reconstructed graft. The strain gauge was then placed with the knee held at $90^{\circ}$ of flexion with no loading using metal barbs and 3 separate 3-0 VICRYL sutures along the sensor length (Fig 1). The gauge was placed nearest to its point of minimal displacement as was practically feasible to facilitate measurement of maximal displacement and strain. This was confirmed visually with live output from the sensor. The knee was then taken through a full range of motion and varus and valgus and rotational loads applied to ensure that the sensor was still within its measurable range without invalid measurements. Gauge placement was adjusted as needed until optimized. Visual inspection confirmed no tibial or femoral impingement.

\section{Biomechanical Testing}

Specimens were loaded onto a custom apparatus with the femur rigidly clamped and the potted tibia secured to a hinged testing frame that accommodates knee flexion from $0^{\circ}$ to $90^{\circ}$, as previously described. ${ }^{17-21}$ Positional data of the tibia relative to the femur were obtained using a 3-dimensional motion tracking system (Optotrak Certus, Northern Digital Inc., Ontario, Canada) with infrared diodes attached to the femur and tibia (Fig 2).

Biomechanical testing was carried out in accordance with previously published work. The tibia was statically loaded with an 88-N anterior drawer force applied to the tibia through an anterior eye bolt attached to the aluminum pot, which was confirmed and recorded using a S-beam load cell (LCCA-100; OMEGA Engineering Inc., Stamford, CT). In addition, a $5 \mathrm{~N}-\mathrm{m}$ internal and external moment was applied to a bolt centered on the distal end of the potted tibia via a torque wrench. Each load condition was held for 15 seconds and motion tracking and strain data were recorded during the last 10 seconds. These loads were repeated at $0^{\circ}, 15^{\circ}, 30^{\circ}, 60^{\circ}$, and $90^{\circ}$ of knee flexion. Afterward, knees were also subjected to a simulated pivot shift maneuver wherein a $7 \mathrm{~N}-\mathrm{m}$ valgus load and $5 \mathrm{~N}-\mathrm{m}$ internal rotation torque were simultaneously applied. While the pivot shift is a composite motion, anterior tibial translation alone without tibial rotation was measured under this loading condition.

The loading sequence was repeated for each specimen in the following conditions: (1) ACL-intact, (2) ACLR

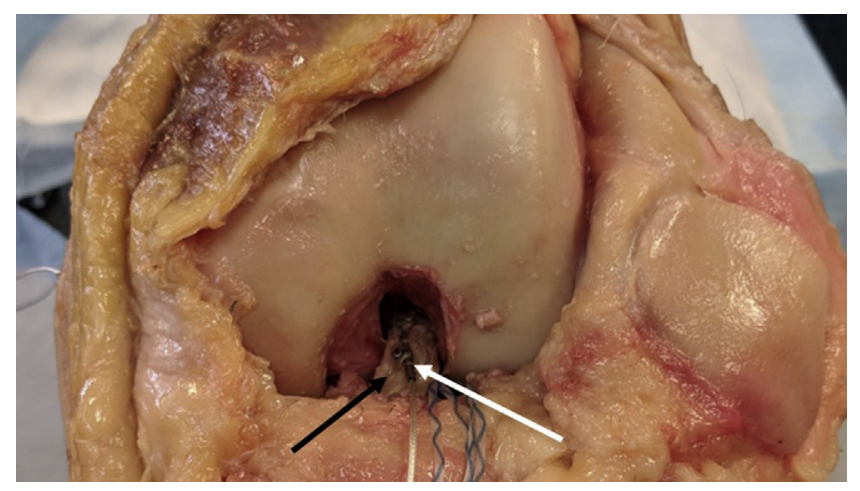

Fig 1. Photograph of mounted left knee specimen before performing meniscal root repair demonstrating the ACL reconstruction with notchplasty and subsequent DVRT placement performed through a median parapatellar arthrotomy. Sutures have been placed for meniscal root repair. Black arrow notes ACL reconstruction graft. White arrow denotes DVRT sensor. (ACL, anterior cruciate ligament; DVRT, differential variable reluctance transducer.) 


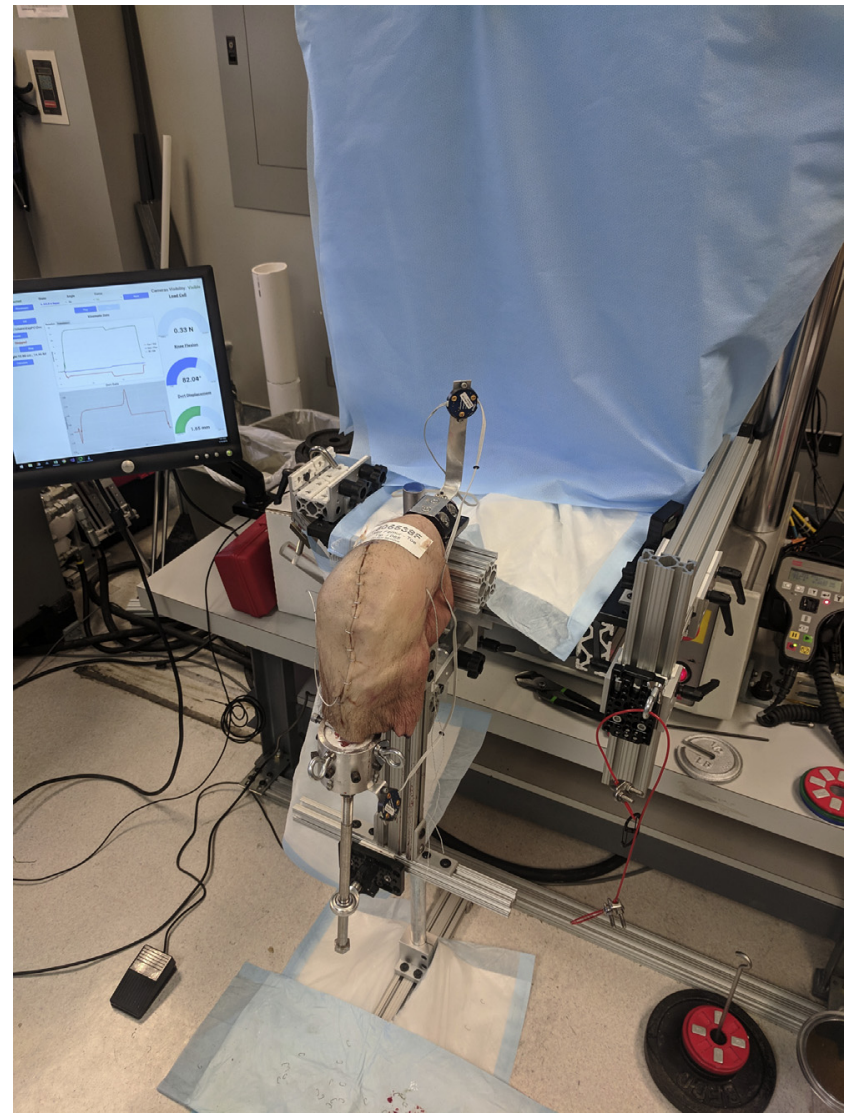

Fig 2. Photograph of a mounted left knee demonstrating the biomechanical testing apparatus.

with LM intact, (3) ACLR with LM posterior root tear, and (4) ACLR with LM root repair. To preserve tissue integrity, all specimens were wrapped in saline-soaked gauze whenever possible.

\section{Strain Measurement}

DVRT displacement and force readings from the load cell were digitally synced. Displacement measurements recorded from the DVRT were used to calculate strain $(\mathcal{E})$, which was defined as the transducer displacement divided by the length of the transducer prior to loading $(\varepsilon=\Delta \mathrm{L} / \mathrm{Lo})$.

\section{Surgical Technique}

A medial parapatellar arthrotomy was performed while carefully preserving the intermeniscal ligament and anterior meniscus attachments before testing the intact state. The menisci, cartilage, and cruciate ligaments were examined for any pathology. Soft-tissue and skin incisions were closed with staples before performing the biomechanical testing protocol for each testing state.

\section{ACL Reconstruction}

All ACL reconstructions were performed by a single sports medicine fellowship-trained surgeon (W.J.U.). Surgeries were performed under direct visualization through an open arthrotomy using the following technique. After the intact state had been tested, the central $10 \mathrm{~mm}$ of the patellar tendon was sharply harvested along with the attached bone plugs from both the patella and tibia using a handheld oscillating saw. Bone plugs were sized to be $10-\times 25-\mathrm{mm}$ in size. Two $2.5-\mathrm{mm}$ holes were drilled at even spacing into the patellar bone plug and two polyblend sutures were passed through the drill holes. A single drill hole was made through the tibial bone block and a single polyblend suture was passed for later graft placement. The graft was then measured and kept in saline soaked gauze until graft placement. The ACL was then sectioned near the native femoral and tibial footprints and carefully removed while preserving the anterior and posterior meniscal attachments and the native posterior cruciate ligament. A 2.4-mm Beath pin was passed through the center of the ACL femoral footprint with the knee in a hyper flexed position and a $10-\mathrm{mm}$ closed-socket femoral tunnel was reamed at $120^{\circ}$ of knee flexion with a $10-\mathrm{mm}$ low-profile reamer to a minimum depth of $25 \mathrm{~mm}$ to simulate the reaming position that would be achieved through an accessory anteromedial arthroscopic portal. Afterward, a tibial ACL drill guide was used to pass a 2.4-mm drill pin through the center of the tibial footprint of the ACL. The guide was initially set at $55^{\circ}$ and adjustments were made to facilitate appropriate tunnel length to prevent tunnel-graft mismatch. The tibial tunnel was then reamed outside-in with a $10-\mathrm{mm}$ diameter cannulated reamer. The ACL graft was then shuttled and fixed in the femur with a $7 \times 20$-mm cannulated interference titanium screw placed in front of the cancellous surface of the plug. The knee was cycled several times under manual graft tension and placed in $0^{\circ}$ of flexion and neutral rotation and fixated with a $9 \times 25$ titanium cannulated interference screw positioned on the anterior aspect of the cancellous portion of the bone plug while applying manual graft tension and a posteriorly directed force on the proximal tibia taking care to avoid varus or valgus stress. Tibial fixation was reinforced by tying the remaining suture limbs over a $3.5-\mathrm{mm}$ cortical screw fixed $1 \mathrm{~cm}$ distally within the tibial cortex.

\section{Posterior Root Tear}

The posterior root of the LM was sectioned with a banana blade near its anatomic attachment under direct visualization approximately $1 \mathrm{~mm}$ posterior and $4 \mathrm{~mm}$ medial to the lateral tibial eminence while taking care to preserve the ACL graft and posterior cruciate ligament. ${ }^{22}$ The meniscofemoral ligaments, both anterior (Humphry) and posterior (Wrisberg) when present, also were sectioned to complete the posterior root injury. The LM was then probed under direct visualization to confirm it was completely mobile from its posterior root attachments. 


\section{Posterior Root Repair}

The LM posterior root repair was performed using a single tunnel transosseous pull-out repair technique. ${ }^{6,7,23}$ The femoral and tibial tunnels for the ACL reconstruction were drilled first as outlined above. Next, a tibial drill guide was used to mark the location for placement of a 2.4-mm guide pin from the anteromedial tibia to the insertion of the posterior root attachment to avoid tunnel convergence. The tunnel was then drilled with a 4-mm cannulated drill. A meniscal suture-passing device was used to place two shuttling sutures near the meniscal free edge to facilitate passage of two 1.3-mm tape sutures. These were placed in a luggage-tag fashion through the root and brought out through the 4-mm tunnel with a looped nitinol suture passer and passed through a four-hole $12-\mathrm{mm}$ round button. The root was reduced to its footprint under direct visualization with manual suture tension and the paired sutures were alternately tied over the button with the knee flexed to $90^{\circ}$.

\section{Statistical Analysis}

An a priori power analysis was calculated $\left(G^{*}\right.$ Power 3.0.10) to determine the number of specimens to include based on previously reported means and standard deviations for anterior tibial translation in an ACLR with posterior LM root injury ${ }^{7}$ and ACL strain before and after a medial collateral ligament injury ${ }^{16}$ confirming a minimum of 8 specimens would be required with $\alpha$ set at 0.05 and power $(1-\beta)$ set at 0.8 . In addition, a post hoc power analysis was conducted to determine the power achieved. A post-hoc power analysis using strain as a primary end point revealed that the study was adequately powered for pivot shift testing $([1-\beta]=0.99)$ but was underpowered for anterior loading $([1-\beta]=0.71)(n=11$, effect size $=0.367$ with an $\alpha$ set at 0.05 ). Statistical analysis was performed using SAS statistical software (version 9.4, SAS Institute, Inc., Cary, NC). After verification of normally distributed data, a 2-way mixed repeated measure analysis of variance model was used to determine the effect of each surgical condition (intact, ACLR, ACLR with root tear, and ACLR with root repair) and each angle of knee flexion on ACL strain and knee kinematics. Tukey-Kramer was used to adjust for multiple comparisons. Significance was set at $P<.05$ and all data are presented as mean \pm standard deviation. ${ }^{7}$

\section{Results}

Of the 14 knees acquired, twelve completed all testing procedures, ( 6 male: 6 female, 7 left and 5 right) with a mean age of 48.9 years ( \pm 6.6 , range: $38-58$ ). Two specimens were excluded: one was not properly secured and rotated within the femoral clamp during testing, and the other experienced graft pullout midway through testing secondary to inadequate tibial fixation related to specimen bone quality. The data from the

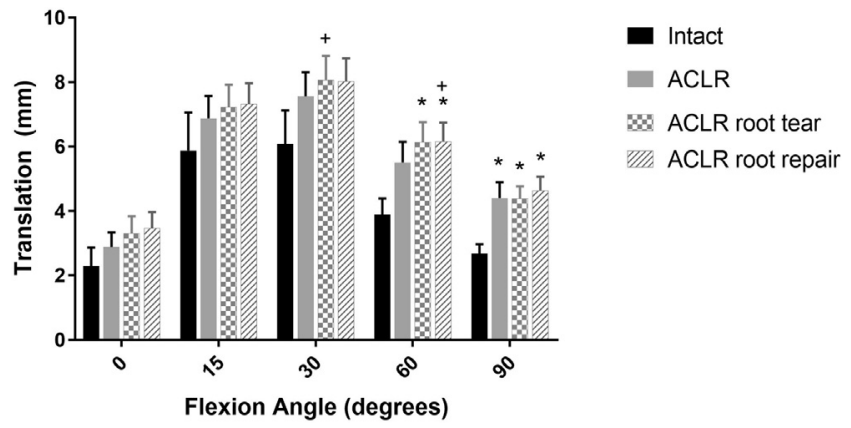

Fig 3. Average anterior tibial translation $( \pm S D)$ as a function of knee flexion angle. Note: * denotes a $P<.05$ when compared with the intact state, + denotes a $P<.05$ when compared with the ACLR state. (ACLR, anterior cruciate ligament reconstruction.)

remaining 12 knees were reviewed and included in the kinematic analysis. The DVRT shifted in one specimen resulting in unreliable data and therefore only eleven specimens were included in the final strain analysis.

\section{Kinematic Data}

\section{Anterior Tibial Translation}

When subjected to an $88 \mathrm{~N}$ anterior load, the average anterior tibial translation of knees with an ACLR and a sectioned LM posterior root complex was significantly increased at $30^{\circ}(\mathrm{ACLR}=7.6 \pm 2.6 \mathrm{~mm}$ vs ACLR $\mathrm{w} /$ tear $=8.1 \pm 2.6 \mathrm{~mm}, P=.04)$ but did not reduce with root repair (Fig 3).

\section{Internal and External Rotation}

When a $5 \mathrm{~N}$-m internal torque was applied to the tibia of knees with an ACLR and a sectioned LM posterior root complex tibial internal rotation significantly increased at $30^{\circ}$ of flexion $\left(\mathrm{ACLR}=23.9 \pm 1.2^{\circ}\right.$ vs ACLR w/tear $\left.=24.7 \pm 1.3^{\circ}, P=.04\right)$ but was not restored with root repair (Fig 4).

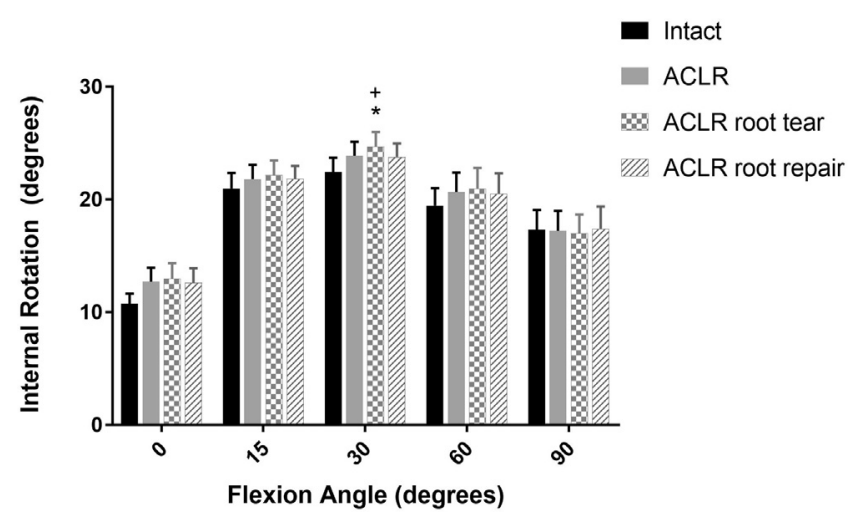

Fig 4. Average $( \pm S D)$ internal rotation as a function of knee flexion. Note: * denotes a $P<.05$ when compared with the intact state, + denotes a $P<0.05$ when compared to the ACLR state. (ACLR, anterior cruciate ligament reconstruction.) 


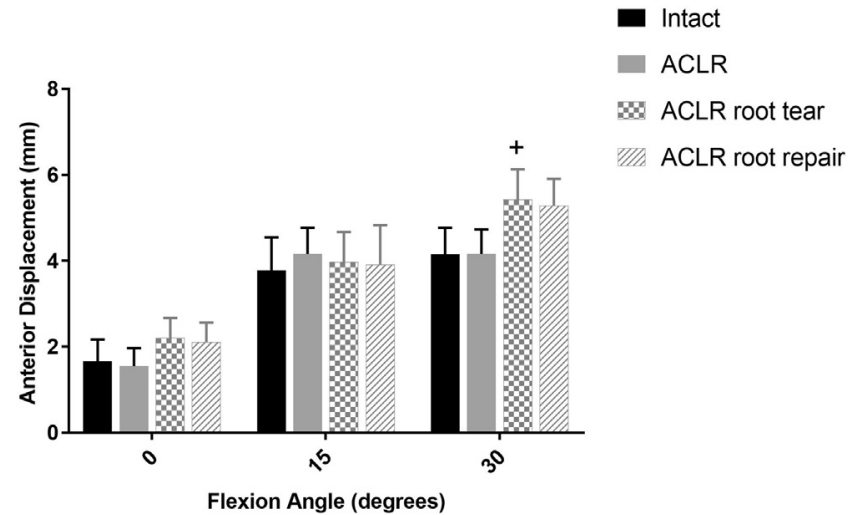

Fig 5. Average $( \pm S D)$ anterior tibial translation measured during simulated pivot shift at $0^{\circ}, 15^{\circ}$, and $30^{\circ}$ of flexion. Note: + denotes $P<.05$ when compared with ACLR. (ACLR, anterior cruciate ligament reconstruction.)

\section{Pivot Shift}

When a simulated pivot shift maneuver was applied to knees with an ACLR and a sectioned LM posterior root complex, anterior tibial translation significantly increased at $30^{\circ}(\mathrm{ACLR}=4.9 \pm 2.3 \mathrm{~mm}$ vs ACLR w/ tear $=4.3 \pm 1.9 \mathrm{~mm}, P=.04)$ but was not restored with root repair (Fig 5).

\section{Strain Data}

\section{Anterior Tibial Translation}

When the tibia was anteriorly loaded to $88 \mathrm{~N}$, sectioning of the LM posterior root complex significantly increased ACL graft strain at $30^{\circ}$ of knee flexion compared with the ACLR state with an intact meniscus $(6.2 \pm 3.3 \%$ vs $4.3 \pm 3.1 \%, P=.01)$ but was not restored with root repair (Fig 6A, Table 1).

\section{Internal and External Rotation}

When a $5 \mathrm{~N}$-m tibial internal torque was applied to the tibia, strain across the ACLR graft increased significantly after sectioning of the LM posterior root complex at $30^{\circ}$ of flexion $(4.5 \pm 3.3 \%$ vs $6.5 \pm 3.3 \%$, $P=.001$ ) (Fig 6B, Tables 2 and 3). Root repair reduced strain to levels that were not significantly different than ACLR with an intact meniscus $(5.5 \pm 3.1 \%, P=.18)$.

\section{Pivot Shift}

When knees were subjected to a simulated pivot shift maneuver, sectioning of the LM posterior root complex significantly increased ACLR graft strain at $15^{\circ}$ $(3.4 \pm 2.0 \%$ vs $4.9 \pm 2.2 \%, P=.04)$ compared to ACLreconstructed knees with an intact meniscus (Fig 6C, Table 4). ACLR graft strain decreased after root repair to $4.6 \pm 3.2 \%$ at $15^{\circ}$ of flexion which was not significantly different than ACLR graft strain with an intact meniscus $(P=.40)$.

\section{Discussion}

The main finding of this time-zero biomechanical study was that injury to the LM posterior root complex in ACL-reconstructed knees increased strain across the ACLR graft when internal torque and pivot shift maneuver was applied to the tibia. Root repair did not significantly reduce graft strain when subjected to loading in this study. LM root tears also increased ACLR graft strain during anterior loading of the knee, although root repair did not successfully mitigate this increase in graft strain. These findings corroborate previous studies that outline the role of the LM posterior root as an important secondary stabilizer and quantify the amount of excess strain placed across ACLR grafts when the LM root is injured. In addition, it suggests that current root repair strategies may have a protective role in minimizing ACLR graft strain, but do not fully restore strain to pretorn levels.

Strain within a ligament or tendon is a measure of elongation or "stretch" in response to a tensile load. Initially, small loads produced large strains as collagen fibers become aligned in what is known as the toe region of the stress-strain curve. As tensile loads increase and all collagen fibers are recruited, the tissue experiences an approximately linear increase in strain dictated by its elastic modulus. This increase in strain continues until relatively large stresses develop in the tissue and the collagen fibers become damaged and fracture leading to failure of the tissue. Studies on the native ACL indicate activities of daily living produce strains of $4 \%$ or less, with failure occurring somewhere
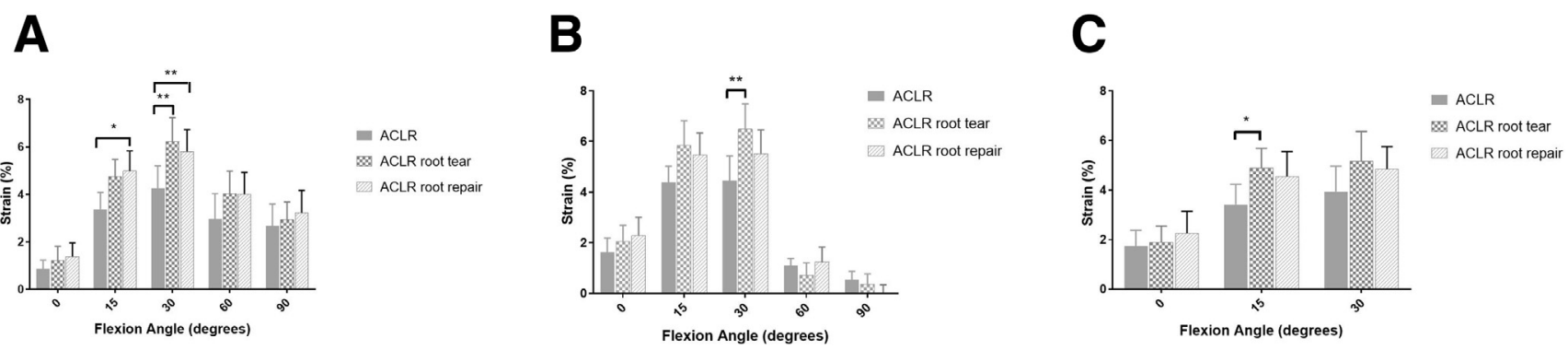

Fig 6. Average $( \pm S D)$ ACL graft strain measured during $(A)$ anterior tibial loading, $(B)$ internal torque, and $(C)$ simulated pivot shift loading as a function of knee flexion. ${ }^{*}$ indicates $P<.05$ and $* *$ indicates $P<.01$. (ACLR, anterior cruciate ligament reconstruction.) 
Table 1. $88 \mathrm{~N}$ Anterior Drawer Force

\begin{tabular}{|c|c|c|c|c|c|}
\hline & $0^{\circ}$ & $15^{\circ}$ & $30^{\circ}$ & $60^{\circ}$ & $90^{\circ}$ \\
\hline \multicolumn{6}{|l|}{ Intact } \\
\hline $\mathrm{ATT}, \mathrm{mm}$ & $2.29 \pm 1.98$ & $5.88 \pm 4.10$ & $6.08 \pm 3.60$ & $3.89 \pm 1.72$ & $2.69 \pm 0.98$ \\
\hline Strain, \% & $0.8 \pm 0.8$ & $4.5 \pm 2.3$ & $4.1 \pm 2.4$ & $2.3 \pm 2.1$ & $1.4 \pm 1.0$ \\
\hline $\mathrm{ATT}, \mathrm{mm}$ & $2.88 \pm 1.58$ & $6.87 \pm 2.40$ & $7.56 \pm 2.61$ & $5.51 \pm 2.19$ & $4.41 \pm 1.68$ \\
\hline Strain, \% & $0.9 \pm 1.2$ & $3.4 \pm 2.4$ & $4.3 \pm 3.1$ & $3.0 \pm 3.5$ & $2.7 \pm 3.1$ \\
\hline \multicolumn{6}{|c|}{ ACLR with tear } \\
\hline \multicolumn{6}{|c|}{ ACLR with repair } \\
\hline ATT, mm & $3.46 \pm 1.76$ & $7.32 \pm 2.25$ & $8.02 \pm 2.46$ & $6.16 \pm 2.01$ & $4.65 \pm 1.46$ \\
\hline Strain, \% & $1.4 \pm 1.9$ & $5.0 \pm 2.8$ & $5.8 \pm 3.1$ & $4.0 \pm 3.0$ & $3.2 \pm 3.1$ \\
\hline
\end{tabular}

NOTE. All values presented as mean \pm standard deviation.

ACLR, anterior cruciate ligament reconstruction; ATT, anterior tibial translation.

above $15 \% .^{24-26}$ While the present study recorded statistically significant differences in strain during certain subsets of simulated knee loading, whether these differences are clinically significant remains to be determined. A post-hoc power analysis demonstrated that this study was adequately powered for strain comparisons during pivot shift loading but was not adequately powered during anterior loading of the tibia. The authors acknowledge the statistical fragility of these conclusions, given the relatively large standard deviations in strain when compared to the absolute increase in strain in these loading conditions. The observed increases in strain experienced under these loading parameters were close to what is believed to be experienced in activities of daily living compared to those that are associated with injury.

While previous studies have theorized that ACLR graft force would increase in the setting of posterior lateral root deficiency, ${ }^{9,10}$ the present study directly measured strain across ACLR grafts in the presence of a root tear and after subsequent repair. Numerous studies have evaluated knee kinematics before and after meniscal root injury and repair..$^{5-10,14,15}$ In 2015,
Shybut et al. ${ }^{9}$ reported that avulsion of the LM posterior root in ACL-deficient knees further destabilized the knee when a pivot shift load was applied to the knee, but found no significant increase in anterior translation when the knee was anteriorly loaded. Frank et al. ${ }^{10}$ corroborated these results and measured a significant increase of approximately $1^{\circ}$ when an internal torque was applied after sectioning the LM root in ACL-intact knees. In 2018, Forkel et al. ${ }^{6}$ evaluated meniscal root injury and repair in ACL-deficient knees and determined that root injury increased rotational laxity and subsequent root repair using a transtibial pullout technique helped restore some internal rotation stability but not to pre-torn levels. Most recently, Tang et al. ${ }^{7}$ evaluated the kinematics of LM root injury in ACLR knees. They reported a significant increase in anterior knee laxity after sectioning the LM posterior root complex that was restored to pre-torn levels after root repair. Also, during simulated pivot shift testing they reported no difference in anterior translation after root injury and that subsequent repair overconstrained the knee. In the same paper, Tang et al. inferred ACLR graft force using a subtractive robotic technique. ${ }^{7}$ Their results

Table 2. 5 N-m Internal Rotation Torque

\begin{tabular}{|c|c|c|c|c|c|}
\hline & $0^{\circ}$ & $15^{\circ}$ & $30^{\circ}$ & $60^{\circ}$ & $90^{\circ}$ \\
\hline \multicolumn{6}{|l|}{ Intact } \\
\hline IR, ${ }^{\circ}$ & $10.74 \pm 3.07$ & $20.97 \pm 4.72$ & $22.42 \pm 4.44$ & $19.46 \pm 5.28$ & $17.31 \pm 6.09$ \\
\hline Strain, \% & $1.9 \pm 1.0$ & $5.2 \pm 3.4$ & $3.3 \pm 1.9$ & $-0.2 \pm 0.8$ & $-0.2 \pm 1.0$ \\
\hline$I R,{ }^{\circ}$ & $12.69 \pm 4.29$ & $21.80 \pm 4.40$ & $23.88 \pm 4.31$ & $20.65 \pm 6.01$ & $17.22 \pm 6.04$ \\
\hline Strain, \% & $1.6 \pm 1.9$ & $4.4 \pm 2.1$ & $4.4 \pm 3.3$ & $1.1 \pm 0.9$ & $0.5 \pm 1.1$ \\
\hline \multicolumn{6}{|c|}{ ACLR with tear } \\
\hline \multicolumn{6}{|c|}{ ACLR with repair } \\
\hline $\mathrm{IR}^{\circ}{ }^{\circ}$ & $12.60 \pm 4.43$ & $21.81 \pm 4.01$ & $23.75 \pm 4.19$ & $20.52 \pm 6.14$ & $17.41 \pm 6.82$ \\
\hline Strain, \% & $2.3 \pm 2.4$ & $5.5 \pm 2.8$ & $5.5 \pm 3.1$ & $1.2 \pm 2.0$ & $0.0 \pm 1.0$ \\
\hline
\end{tabular}

NOTE. All values presented as mean \pm standard deviation.

ACLR, anterior cruciate ligament reconstruction; IR, internal rotation. 
Table 3. 5 N-m External Rotation Torque

\begin{tabular}{|c|c|c|c|c|c|}
\hline & $0^{\circ}$ & $15^{\circ}$ & $30^{\circ}$ & $60^{\circ}$ & $90^{\circ}$ \\
\hline \multicolumn{6}{|l|}{ Intact } \\
\hline 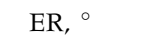 & $11.67 \pm 4.34$ & $18.75 \pm 4.93$ & $19.53 \pm 4.81$ & $22.42 \pm 5.36$ & $24.85 \pm 5.11$ \\
\hline Strain, \% & $-1.0 \pm 1.6$ & $1.4 \pm 2.3$ & $0.5 \pm 2.9$ & $-1.9 \pm 2.6$ & $-3.3 \pm 2.3$ \\
\hline \multicolumn{6}{|l|}{ ACLR } \\
\hline 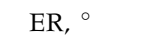 & $11.77 \pm 5.22$ & $19.54 \pm 4.60$ & $20.31 \pm 5.08$ & $22.28 \pm 5.36$ & $24.93 \pm 4.71$ \\
\hline Strain, \% & $0.0 \pm 1.2$ & $0.8 \pm 2.9$ & $1.2 \pm 4.6$ & $-2.9 \pm 2.8$ & $-4.6 \pm 3.4$ \\
\hline \multicolumn{6}{|c|}{ ACLR with tear } \\
\hline 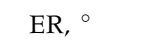 & $12.16 \pm 5.57$ & $18.72 \pm 4.56$ & $21.08 \pm 6.06$ & $22.58 \pm 6.10$ & $25.26 \pm 5.03$ \\
\hline Strain, \% & $0.5 \pm 1.8$ & $1.5 \pm 3.1$ & $1.5 \pm 4.7$ & $-3.1 \pm 2.8$ & $-5.3 \pm 3.9$ \\
\hline \multicolumn{6}{|c|}{ ACLR with repair } \\
\hline $\mathrm{ER},{ }^{\circ}$ & $12.65 \pm 5.65$ & $19.21 \pm 4.85$ & $21.55 \pm 6.08$ & $22.43 \pm 6.09$ & $24.19 \pm 5.52$ \\
\hline Strain, \% & $0.4 \pm 2.1$ & $1.7 \% \pm 3.1$ & $0.6 \% \pm 5.0$ & $-3.5 \% \pm 3.1$ & $-5.5 \% \pm 3.0$ \\
\hline
\end{tabular}

NOTE. All values presented as mean \pm standard deviation

ACLR, anterior cruciate ligament reconstruction; ER, external rotation.

indicate that a LM root tear does not increase force on an ACLR graft; rather, subsequent repair increases ACLR strain to levels greater than an ACLR with an intact meniscus, most significantly at higher angles of flexion.

The findings of this study are similar to Tang et al. as we both demonstrate an increase in anterior laxity after sectioning the LM posterior root. The present study differs from Tang el al. in the additional finding that an increase in joint laxity corresponds to a similar increase in ACLR strain. In addition, Tang did not measure changes in response to rotational loads, although our post-injury kinematics are similar to the $1^{\circ}$ increase in rotational instability reported by Forkel et al. ${ }^{6}$ Similar to Forkel et al, the present study was unable to demonstrate that root repair significantly restored knee stability.

Not all injuries to the posterior root complex in the setting of ACL injury are functionally the same. Several authors have proposed morphological classifications of these injuries to determine the best method of treatment differentiating between partial and complete radial tears and those with obliquity or tear

Table 4. Pivot Shift

\begin{tabular}{lccc}
\hline & $0^{\circ}$ & $15^{\circ}$ & $30^{\circ}$ \\
\hline Intact & & & \\
$\quad$ ATT, mm & $1.27 \pm 1.93$ & $4.42 \pm 3.75$ & $4.10 \pm 2.78$ \\
$\quad$ Strain, \% & $2.1 \pm 1.8$ & $4.9 \pm 2.5$ & $3.7 \pm 2.6$ \\
ACLR & & & \\
$\quad$ ATT, mm & $1.80 \pm 1.36$ & $4.57 \pm 1.93$ & $4.16 \pm 1.87$ \\
$\quad$ Strain, \% & $1.7 \pm 2.1$ & $3.4 \pm 2.0$ & $3.9 \pm 2.7$ \\
ACLR with Tear & & & \\
$\quad$ ATT, mm & $1.91 \pm 1.51$ & $5.09 \pm 2.23$ & $4.70 \pm 2.38$ \\
$\quad$ Strain, \% & $1.9 \pm 2.2$ & $4.9 \pm 2.2$ & $5.2 \pm 2.6$ \\
ACLR with Repair & & & \\
$\quad$ ATT, mm & $1.61 \pm 1.47$ & $4.27 \pm 2.97$ & $4.09 \pm 2.17$ \\
Strain, \% & $2.3 \pm 2.9$ & $4.6 \pm 3.2$ & $4.9 \pm 3.3$ \\
\hline
\end{tabular}

NOTE. All values presented as mean \pm standard deviation.

ACLR, anterior cruciate ligament reconstruction; ATT, anterior tibial translation. extension. ${ }^{22,27}$ The classification by Forkel et al. ${ }^{3}$ Click or tap here to enter text. accounted for additional injury to the meniscofemoral ligaments as their absence has been noted to alter tibiofemoral contact pressures and provide additive rotational instability in several biomechanical studies. ${ }^{6,10,14,28}$ This is an important clinical consideration as one would presume that a complete radial root tear with an injured meniscofemoral ligament would behave differently after transtibial root repair than a complete root tear alone. While the body of literature is vast and difficult to search, very little is known regarding the comparative laxity that may be induced by the two injury complexes. Thus, additional research that identifies the specific role of the meniscofemoral ligaments in modifying ACLR graft strain under translational and rotational loading is needed.

When comparing the present study to the work of Tang et al. ${ }^{7}$ and Forkelet al., ${ }^{6}$ Click or tap here to enter text., important differences in the testing apparatus should be noted. Tang and Forkel both used an axially preloaded robotic testing apparatus which is believed to better recreate physiologic knee loading and motion. A robotic testing apparatus also allows for the measurement of the pivot shift loading as a composite rotatory and translational motion as opposed to a simplified linear motion as measured in the present study. The authors acknowledge the inherent limitations of a more traditional testing apparatus without any measured axial preloading.

\section{Limitations}

As with all biomechanical studies, the data presented in this study should be viewed considering several important limitations. First, while there was an appropriate a priori and post-hoc power analysis, the relatively large standard deviations and correspondingly small relative increases in strain measurements do limit the ability to confidently declare the findings as clinically significant. While care was exercised to ensure 
reliable and stable placement of the strain gauge, there is a degree of variability when placing DVRT sensors onto soft tissue. ${ }^{29,30}$ Some of this reported variability is due to structural variations along the length of native ligamentous tissue that are not applicable to the prepared single bundle patellar tendon autografts used in this study. In addition, this study represents testing under a time-zero state without the ability to account for additional effect that may arise with normal biological healing. Finally, there are several experimental design limitations. First, no axial load was applied. Second, the apparatus did not measure the rotational component of pivot shift motion. Third, the order of flexion angle tested was intentionally not randomized to minimize error and motion associated with placement and replacement of the DVRT strain gauge in a randomized design. This may have contributed to variations in laxity, although quasi-static testing techniques, physiologic nondestructive loading, and careful observation for any increase in motion caused by tissue fatigue likely minimized this effect. Finally, it is important to that the apparatus used was different that other studies in that it was not performed on a robotic testing apparatus.

\section{Conclusions}

This cadaveric biomechanical study suggests injury to the LM posterior root complex increases rotational and anterior laxity of the knee and places increased strain across reconstructed ACL grafts. Subsequent root repair did not result in a statistically significant reduction in strain.

\section{Acknowledgments}

The authors thank Jeff Gornbein, M.S., Dr.P.H., of UCLA David Geffen School of Medicine for his effort and assistance with the statistical portion of the data analysis.The medical illustrations were provided courtesy of Arthrex.

\section{References}

1. Smet AAD, Blankenbaker DG, Kijowski R, Graf BK, Shinki K. MR diagnosis of posterior root tears of the lateral meniscus using arthroscopy as the reference standard. Am J Roentgenol 2009; 192:480-486.

2. Brody JM, Lin HM, Hulstyn MJ, Tung GA. Lateral meniscus root tear and meniscus extrusion with anterior cruciate ligament tear. Radiology 2006;239:805-810.

3. Forkel P, Reuter S, Sprenker F, et al. Different patterns of lateral meniscus root tears in ACL injuries: Application of a differentiated classification system. Knee Surg Sports Traumatol Arthrosc 2014;23:112-118.

4. Matheny LM, Ockuly AC, Steadman JR, LaPrade RF. Posterior meniscus root tears: associated pathologies to assist as diagnostic tools. Knee Surg Sports Traumatol Arthrosc 2015;23:1-5.
5. Praz C, Vieira TD, Saithna A, et al. Risk factors for lateral meniscus posterior root tears in the anterior cruciate ligament-injured knee: An epidemiological analysis of 3956 patients from the SANTI study group. Am J Sports Med 2019;105:1-8.

6. Forkel P, Deimling C von, Lacheta L, et al. Repair of the lateral posterior meniscal root improves stability in an ACL-deficient knee. Knee Surg Sports Traumatol Arthrosc 2018;26:2302-2309.

7. Tang X, Marshall B, Wang JH, et al. Lateral meniscal posterior root repair with anterior cruciate ligament reconstruction better restores knee stability. Am J Sports Med 2018;47:1-7.

8. Minami T, Muneta T, Sekiya I, et al. Lateral meniscus posterior root tear contributes to anterolateral rotational instability and meniscus extrusion in anterior cruciate ligament-injured patients. Knee Surg Sports Traumatol Arthrosc 2017;26:1-8.

9. Shybut TB, Vega CE, Haddad J, et al. Effect of lateral meniscal root tear on the stability of the anterior cruciate ligament-deficient knee. Am J Sports Med 2015;43:1-7.

10. Frank JM, Moatshe G, Brady AW, et al. Lateral meniscus posterior root and meniscofemoral ligaments as stabilizing structures in the ACL-deficient knee: A biomechanical study. Orthop J Sports Med 2017;5:232596711769575232596711769577.

11. Kamatsuki Y, Furumatsu T, Fujii M, et al. Complete tear of the lateral meniscus posterior root is associated with meniscal extrusion in anterior cruciate ligament deficient knees. J Orthop Res 2018;36:1894-1900.

12. Shelbourne KD, Roberson TA, Gray T. Long-term evaluation of posterior lateral meniscus root tears left in situ at the time of anterior cruciate ligament reconstruction. Am J Sports Med 2011;39:1-5.

13. Perez-Blanca A, Espejo-Baena A, Trujillo D, et al. Comparative biomechanical study on contact alterations after lateral meniscus posterior root avulsion, transosseous reinsertion, and total meniscectomy. Arthroscopy 2016;32:624-633.

14. Forkel P, Herbort M, Sprenker F, Metzlaff S, Raschke M, Petersen W. The biomechanical effect of a lateral meniscus posterior root tear with and without damage to the meniscofemoral ligament: Efficacy of different repair techniques. Arthroscopy 2014;30:833-840.

15. LaPrade CM, Jansson KS, Dornan G, Smith SD, Wijdicks CA, LaPrade RF. Altered tibiofemoral contact mechanics due to lateral meniscus posterior horn root avulsions and radial tears can be restored with in situ pull-out suture repairs. J Bone Joint Surg 2014;96: 471-479.

16. Mancini EJ, Kohen R, Esquivel AO, Cracchiolo AM, Lemos SE. Comparison of ACL strain in the MCL-deficient and MCL-reconstructed knee during simulated landing in a cadaveric model. Am J Sports Med 2017;45:1-5.

17. Kremen TJ, Polakof LS, Rajaee SS, Nelson TJ, Metzger MF. The effect of hamstring tendon autograft harvest on the restoration of knee stability in the setting of concurrent anterior cruciate ligament and medial collateral ligament injuries. Am J Sports Med 2017;46: $163-170$ 
18. Trentacosta N, Pace JL, Metzger M, et al. Biomechanical evaluation of pediatric anterior cruciate ligament (ACL) reconstruction techniques with and without the anterolateral ligament (ALL). J Pediatr Orthoped 2020;40:8-16.

19. Welch T, Keller T, Maldonado R, Metzger M, Mohr K, Kvitne R. The effect of a dynamic PCL brace on patellofemoral compartment pressures in PCL-and PCL/ PLC-deficient knees. J Exp Orthop 2017;4:17.

20. Kennedy A, Coughlin DG, Metzger MF, et al. Biomechanical evaluation of pediatric anterior cruciate ligament reconstruction techniques. Am J Sports Med 201 1;39:964-971.

21. Chahla J, Nelson T, Dallo I, et al. Anterior cruciate ligament repair versus reconstruction: A kinematic analysis. Knee 2020;27:334-340.

22. LaPrade CM, James EW, Cram TR, Feagin JA, Engebretsen L, LaPrade RF. Meniscal root tears: A classification system based on tear morphology. Am J Sports Med 2015;43:363-369.

23. Frank JM, Liechti DJ, Anavian J, Hackett TR. A transosseous bone bridge repair for posterior horn meniscal root tears during anterior cruciate ligament reconstruction. Arthrosc Tech 2017;6:e239-e243.

24. Beynnon BD, Fleming BC. Anterior cruciate ligament strain in-vivo: A review of previous work. J Biomech 1998;31:519-525.
25. Fleming BC, Beynnon BD. In vivo measurement of ligament/tendon strains and forces: A review. Ann Biomed Eng 2004;32:318-328.

26. Butler DL, Guan Y, Kay MD, Cummings JF, Feder SM, Levy MS. Location-dependent variations in the material properties of the anterior cruciate ligament. J Biomech 1992;25:511-518.

27. Ahn JH, Lee YS, Yoo JC, Chang MJ, Park SJ, Pae YR. Results of arthroscopic all-inside repair for lateral meniscus root tear in patients undergoing concomitant anterior cruciate ligament reconstruction. Arthroscopy 2010;26:67-75.

28. Ohori T, Mae T, Shino K, et al. Complementary function of the meniscofemoral ligament and lateral meniscus posterior root to stabilize the lateral meniscus posterior horn: A biomechanical study in a porcine knee model. Orthop J Sports Med 2019;7:1-7.

29. Beynnon B, Howe JG, Pope MH, Johnson RJ, Fleming BC. The measurement of anterior cruciate ligament strain in vivo. Int Orthop 1992;16:1-2.

30. Bates NA, Nesbitt RJ, Shearn JT, Meyer GD, Hewett TE. Relative strain in the anterior cruciate ligament and medial collateral ligament during simulated jump landing and sidestep cutting tasks. Am J Sports Med 2015;43:1-11. 